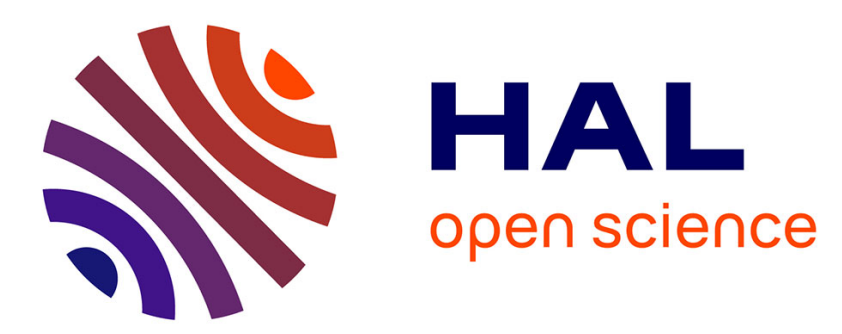

\title{
Effets de la recombinaison de surface et des conditions d'excitation sur les réponses de photoconductivité transitoire
}

\author{
S. Rebiai, D. Bielle-Daspet
}

\section{To cite this version:}

S. Rebiai, D. Bielle-Daspet. Effets de la recombinaison de surface et des conditions d'excitation sur les réponses de photoconductivité transitoire. Revue de Physique Appliquée, 1986, 21 (9), pp.545-556. 10.1051/rphysap:01986002109054500 . jpa-00245472

HAL Id: jpa-00245472

https://hal.science/jpa-00245472

Submitted on 1 Jan 1986

HAL is a multi-disciplinary open access archive for the deposit and dissemination of scientific research documents, whether they are published or not. The documents may come from teaching and research institutions in France or abroad, or from public or private research centers.
L'archive ouverte pluridisciplinaire $\mathbf{H A L}$, est destinée au dépôt et à la diffusion de documents scientifiques de niveau recherche, publiés ou non, émanant des établissements d'enseignement et de recherche français ou étrangers, des laboratoires publics ou privés. 
Classification

Physics Abstracts

$07.50-72.40$

\title{
Effets de la recombinaison de surface et des conditions d'excitation sur les réponses de photoconductivité transitoire
}

\author{
S. Rebiai et D. Bielle-Daspet \\ Laboratoire d'Automatique et d'Analyse des Systèmes du Centre National de la Recherche Scientifique, \\ 7, avenue du Colonel Roche, 31077 Toulouse Cedex, France
}

(Reçu le 10 février 1986, révisé le 28 avril 1986, accepté le 15 mai 1986)

\begin{abstract}
Résumé - Les expressions théoriques de la distribution $\Delta n(x, t)$ de l'excès de porteurs et de sa valeur intégrée $\Delta \mathcal{N}(t)$ sont successivement traitées pour des impulsions lumineuses rectangulaires et d'allure gaussienne. L'analyse est menée de façon à mettre en relief les effets respectifs de la vitesse de recombinaison en surface $S$, de la constante de diffusion $D$ et de la durée de vie volumique $\tau_{\mathbf{B}}$ des porteurs dans un échantillon, ainsi que de la durée $\delta$ et du coefficient d'absorption $\alpha$ de l'impulsion lumineuse. Ces cinq paramètres, ajoutés à l'épaisseur $W$ de l'échantillon, sont pris en compte pour discuter des conditions sous lesquelles les valeurs de $S$ et $\tau_{\mathrm{B}}$ peuvent être obtenues à partir des mesures du rapport $K\left(t_{+}, t_{-}\right)$, entre les pentes de part et d'autre du maximum, et de la constante de temps de décroissance $\tau$ de réponses $\Delta \mathcal{N}(t)$. L'illustration numérique est faite pour des cas types d'échantillons de silicium (substrats : $D=34 \mathrm{~cm}^{2} / \mathrm{s}, \tau_{\mathrm{B}}=1 \mu \mathrm{s}$; couches : $\left.D=1 \mathrm{~cm}^{2} / \mathrm{s}, \tau_{\mathrm{B}}=40 \mathrm{~ns}\right)$ et d'excitation lumineuse $(\delta=0,4$ à $100 \mathrm{~ns} ; \lambda=1,06,0,91,0,69$ ou $0,51 \mu \mathrm{m}$; valeurs numériques de $\Delta n, \mathcal{N}$ calculées pour $g_{0}=10^{21} \mathrm{~cm}^{-3} \mathrm{~s}^{-1}$ ).
\end{abstract}

\begin{abstract}
The theoretical expressions of the excess carrier distribution $\Delta n(x, t)$ and its integrated value $\Delta \mathcal{N}(t)$ are successively considered for rectangular or Gaussian light pulses. The analysis deals with the changes in $\Delta n(x, t)$ and $\Delta \mathcal{N}(t)$ with the carrier surface recombination velocity $S$, diffusion coefficient $D$ and bulk lifetime $\tau_{\mathrm{B}}$, as well as light pulse duration $\delta$ and absorption coefficient $\alpha$. These parameters and the sample thickness $W$ are taken into account in the discussion of the conditions under which the $S$ and $\tau_{\mathrm{B}}$ values may be obtained using the negative ratio $K\left(t_{+}, t_{-}\right)$of the slopes, just before and after the maximum, and the decay time constant $\tau$ of the $\Delta \mathcal{N}(t)$ responses. Numerical calculations are given for typical examples of silicon samples (substrates : $D=34 \mathrm{~cm}^{2} / \mathrm{s}$, $\tau_{\mathrm{B}}=1 \mu \mathrm{s} ;$ layers $\left.: D=1 \mathrm{~cm}^{2} / \mathrm{s}, \tau_{\mathrm{B}}=40 \mathrm{~ns}\right)$ and light pulses $(\delta=0.4$ to $100 \mathrm{~ns}$; wavelengths $\lambda=1.06,0.91,0.69$ and $0.51 \mu \mathrm{m}$; numerical values of $\Delta n, \mathcal{N}$ calculated for $\left.g_{0}=10^{21} \mathrm{~cm}^{-3} \mathrm{~s}^{-1}\right)$.
\end{abstract}

\section{Introduction.}

La mesure de la durée de vie des porteurs dans un échantillon semiconducteur, à partir de la décroissance des réponses de photoconductivité transitoire, relève des techniques classiques et directes de mesures, bien adaptées au suivi de cette durée de vie lors du processus technologique d'élaboration d'un composant [1]. Toutefois, les durées de vie mesurées par ces techniques sont des durées de vie effectives $\tau$ qui peuvent être délicates à interpréter en terme des valeurs respectives de la vitesse de recombinaison en surface $S$ et de la durée de vie volumique $\tau_{\mathbf{B}}$ des porteurs dans l'échantillon. Ceci, par suite de la variation des pertes de porteurs aux surfaces en fonction des conditions expérimentales. Ces pertes varient, en effet, à la fois avec l'échantillon (état $S$ des surfaces, valeur relative de l'épaisseur $W$ et de la longueur de diffusion $L$ des porteurs) et avec la durée $\delta$ et la profondeur de pénétration $1 / \alpha$ de l'excitation lumineuse.
En contre-partie, le contrôle des cinétiques de montée et de décroissance de réponses de photoconductivité transitoire $\left({ }^{1}\right)$, induites par des impulsions de lumière relativement ou peu absorbées $(1 / \alpha \ll$ ou $\sim W)$ et brèves $(\delta \ll \tau)$, offre la possibilité d'atteindre les valeurs respectives de $\tau_{\mathbf{B}}$ et $S$.

Le premier but de l'analyse qui suit est de préciser sous quelles conditions expérimentales $\left(S, D, \tau_{\mathrm{B}}, \delta, \alpha\right)$ la distribution d'un excès de porteurs tend à se trouver dominée soit par les pertes en surface $(S)$, soit par les pertes en volume $\left(\tau_{\mathrm{B}}\right)$ de l'échantillon. Son second but est de mettre en relief quelles conditions d'excitation sont par suite nécessaires pour atteindre les valeurs mêmes de $S$ et $\tau_{\mathrm{B}}$, dans un échantillon donné, et selon quelles méthodes les réponses de photoconductivités transitoire peuvent alors être utilisées.

( $\left.{ }^{1}\right)$ Il peut en être de mềme des réponses de photoluminescences. 
Pour cela, nous avons repris l'approche théorique décrite dans les références $[2,3]$ et nous avons étudié les effets relatifs des conditions d'excitation $(\delta, \alpha)$ et des pertes de porteurs $\left(S, \tau_{\mathrm{B}}\right)$ sur la distribution $\Delta n(x, t)$ et la valeur intégrée $\Delta \mathcal{N}(t)$ de l'excès de porteurs générés. Pour le traitement numérique de cette étude, nous nous sommes intéressées à des castypes de sources lumineuses (diode laser $\mathrm{AsGa}$; laser au rubis, au néodyme ou à l'argon) et à la comparaison d'échantillons de silicium dont les caractéristiques relèvent de celles de films $\left(D=1 \mathrm{~cm}^{2} / \mathrm{s}, \tau_{\mathrm{B}}=40 \mathrm{~ns}\right)$ ou de substrats $\left(D=34 \mathrm{~cm}^{2} / \mathrm{s}, \tau_{\mathrm{B}}=1 \mu \mathrm{s}\right)$. L'application de ce travail est essentiellement discutée dans le sens de son utilisation au suivi technologique de composants du type composants de puissance.

Les paragraphes 2 et 3 traitent du cas d'impulsions lumineuses rectangulaires : le paragraphe 2 donne les résultats obtenus pour la distribution $\Delta n(x, t)$ de l'excès de porteurs, et leur discussion; le paragraphe 3 est consacré à la valeur intégrée $\Delta \mathcal{N}(t)$ de cet excès et met en évidence le rôle des différents paramètres $\left(\alpha, \delta\right.$, et $\left.S, \tau_{\mathrm{B}}, D\right)$ sur ce qui est par la suite appelé le rapport de pentes $K$. Dans le paragraphe 4, les résultats précédents sont utilisés pour traiter du cas d'impulsions lumineuses d'allure gaussienne. Les conclusions de cette analyse sont données au paragraphe 5.

2. Effets relatifs de $S, \tau_{\mathrm{B}}, \delta$ et $\alpha$ sur la distribution $\Delta n(x, t)$ de l'excès de porteurs dans le cas d'une impulsion excitatrice rectangulaire.

2.1 HYPOTHÈSES DE BASE, EXPRESSIONS ANALYTIQUES ET CALCULS NUMÉRIQUES. - L'analyse de base sup- pose un échantillon semiconducteur unidimensionnel $(l . \mathcal{L} \gg W)$ homogène dont l'épaisseur $x=W$ est grande devant la profondeur $1 / \alpha$ de pénétration de la lumière excitatrice utilisée. Ainsi, nous assimilerons, dans ce qui suit, l'échantillon à un échantillon homogène semi-infini.

Lorsque l'échantillon est éclairé - impulsion monochromatique, rectangulaire, brève (durée $\delta \ll \tau_{\mathrm{B}}$ ) et incident sur la face $x=0-$ l'excès de porteurs se trouve généré dans une zone limitée, proche de la surface. La distribution $\Delta n(x, t)$ de porteurs injectés est donc simultanément affectée par les pertes $S$ à la surface de l'échantillon et par la diffusion vers l'intérieur du volume où les porteurs se recombinent avec une durée de vie $\tau_{\mathrm{B}}$. L'expression de $\Delta n(x, t)$ est une fonction des quatre paramètres $\alpha, \delta, S$ et $\tau_{\mathbf{B}}$ qui définissent les conditions expérimentales, et s'obtient par la résolution de l'équation de continuité (1) compte tenu de la condition aux limites (2) :

$$
\frac{\partial n}{\partial t}(x, t)=D \frac{\partial^{2} n(x, t)}{\partial x^{2}}-\frac{n(x, t)}{\tau_{\mathbf{B}}}+g(x, t)
$$

avec :

$$
g(x, t)=g_{0} \mathrm{e}^{-\alpha x} \quad \text { si } \quad 0<t<\delta
$$

et

$$
\left.D \frac{\partial n}{\partial x}\right|_{x=0}=\operatorname{Sn}(0, t)
$$

Utilisant les transformées de Laplace de ces deux relations, on retrouve l'expression de $\Delta n(x, t)$ citée dans la référence [2] :

$$
\begin{aligned}
& \Delta n^{*}(x, t)=\frac{g_{0} L^{3}}{2 D}\left(\alpha+\frac{S}{D}\right) \mathrm{e}^{-t / \tau_{\mathbf{B}}}\left[\frac { 1 } { ( \alpha ^ { 2 } L ^ { 2 } - 1 ) } \left(\left\{\frac{\mathrm{e}^{-x / L}}{\left(\frac{S L}{D}+1\right)} \cdot \operatorname{erfc}\left(\frac{x}{2 \sqrt{D t}}-\sqrt{\frac{t}{\tau_{\mathbf{B}}}}\right)+\right.\right.\right. \\
& \left.+\frac{\mathrm{e}^{x / L}}{\left(\frac{S L}{D}-1\right)} \operatorname{erfc}\left(\frac{x}{2 \sqrt{D t}}+\sqrt{\frac{t}{\tau_{\mathbf{B}}}}\right)\right\} \cdot \mathrm{e}^{t / \tau_{\mathbf{B}}}-\left\{\frac{\mathrm{e}^{-\alpha x}}{\left(\frac{S L}{D}+\alpha L\right)} \operatorname{erfc}\left(\frac{x}{2 \sqrt{D t}}-\alpha L \sqrt{\frac{t}{\tau_{\mathbf{B}}}}\right)+\right. \\
& \left.\left.\left.+\frac{\mathrm{e}^{\alpha x}}{\left(\frac{S L}{D}-\alpha L\right)} \operatorname{erfc}\left(\frac{x}{2 \sqrt{D t}}+\alpha L \sqrt{\frac{t}{\tau_{\mathrm{B}}}}\right)\right\} \mathrm{e}^{\alpha^{2} L^{2} t \tau_{\mathrm{B}}}\right)-2\left(\frac{S L}{D}\right) \frac{\mathrm{e}^{\frac{S x}{D}} \operatorname{erfc}\left(\frac{x}{2 \sqrt{D t}}+\frac{S L}{D} \sqrt{\frac{t}{\tau_{\mathrm{B}}}}\right)}{\left\{\alpha^{2} L^{2}-\left(\frac{S L}{D}\right)^{2}\right\}\left\{\left(\frac{S L}{D}\right)^{2}-1\right\}} \mathrm{e}^{\left(\frac{S L}{D}\right)^{2} t \tau_{\mathrm{B}}}\right] \\
& +\frac{g_{0} \tau_{\mathrm{B}}}{\left(1+\alpha^{2} L^{2}\right)} \mathrm{e}^{-\alpha x}\left[1-\mathrm{e}^{\left(\alpha^{2} L^{2}-1\right) t \tau_{\mathbf{B}}}\right]
\end{aligned}
$$

avec :

$$
\Delta n(x, t)=\Delta n^{*}(x, t) \text { pour } t<\delta
$$

et :

$\Delta n(x, t)=\Delta n^{*}(x, t)-\Delta n^{*}(x, t-\delta)$ pour $t \geqslant \delta$.
Nous avons effectué le calcul numérique de l'expression (3) ci-dessus à l'aide d'un programme basic HP 9845 C.

Par ce calcul, il est possible d'obtenir les variations de $\Delta n(x, t)$ en s'intéressant à l'évolution, soit des profils 
$\Delta n(x)$ à différents temps $t$, soit de l'excès $\Delta n(t)$ en différents points $x$ du matériau.

Notons qu'un tel calcul nécessite d'approcher au mieux la fonction erreur complémentaire erfc $(y)$. Nous avons utilisé pour cela les approximations suivantes [4] :

$$
\begin{array}{llll}
\operatorname{erf}(y)=\left[1-\exp \left(-4 y^{2} / \pi\right)\right]^{1 / 2} & \text { pour } & y<3,5 \\
\operatorname{erf}(y)=\left[1-(1 / 2) \exp \left(-4 y^{2} / \pi\right)\right] & \text { pour } & y \geqslant 3,5
\end{array}
$$

avec :

$$
\operatorname{erfc}(y)=1-\operatorname{erf}(y) \quad \text { et } \operatorname{erfc}(-y)=2-\operatorname{erfc}(y)
$$

qui donnent une allure correcte pour $\operatorname{erfc}(y)$.

\subsection{EFFETS RELATIFS DE L'EXCITATION, LA DIFFUSION} ET LA RECOMBINAISON DES PORTEURS SUR LES PROFILS $\Delta n(x, t)$ DE PORTEURS INJECTÉs. - Afin d'illustrer les effets relatifs des conditions d'excitation $\alpha, \delta$ et de recombinaison de surface $S$ ou de volume $\tau_{\mathbf{B}}$ sur la distribution de l'excès $\Delta n(x, t)$ de porteurs, nous avons appliqué le calcul numérique de l'expression (3) à un ensemble de cas typiques d'échantillons et de sources lumineuses. Les résultats consignés sur les figures 1 et 2 permettent de comparer les évolutions $\Delta n(x)$, avec $t$ comme paramètre, des profils qui sont créés par diverses sources lumineuses dans un même film $\left(D=1 \mathrm{~cm}^{2} / \mathrm{s}, \tau_{\mathrm{B}}=40 \mathrm{~ns}\right)$, ou un même substrat $\left(D=34 \mathrm{~cm}^{2} / \mathrm{s}, \tau_{\mathrm{B}}=1 \mu \mathrm{s}\right)$, de silicium. Ces résultats ont été obtenus pour des vitesses de recombinaison $S=10^{2}$ ou $10^{4} \mathrm{~cm} / \mathrm{s}$ et des impulsions lumineuses rectangulaires de durée $\delta=0,4$ ou 60 ns. Les longueurs d'onde considérées correspondent :

- ̀̀ un laser argon $(\lambda=0,51 \mu \mathrm{m}, \alpha=1,2$ $\times 10^{4} \mathrm{~cm}^{-1}$; Fig. 1a) ou rubis $(\lambda=0,69 \mu \mathrm{m}, \alpha=$ $2200 \mathrm{~cm}^{-1}$; Fig. 1b),

- ou à une diode laser à l'AsGa $(\lambda=0,91 \mu \mathrm{m}$, $\alpha=330 \mathrm{~cm}^{-1}$; Figs. $1 \mathrm{c}$ et $\left.2 \mathrm{a}\right)$,

- ou à un laser au néodyme $(\lambda=1,06 \mu \mathrm{m}, \alpha=$ $30 \mathrm{~cm}^{-1}$; Figs. 1d et 2b).

De l'ensemble des résultats illustrés sur les figures 1 et 2 , trois remarques peuvent être dégagées :

1) La première porte sur l'effet de $S$ : quelle que soit la longueur d'onde de l'excitation utilisée, la distribution de porteurs $\Delta n(x)_{t}$ passe par son maximum à une profondeur $x_{\max }$ qui croît avec la valeur des pertes aux surfaces $S$. De plus, l'effet de $S$ sur cette distribution $\Delta n(x)_{t}$ et sur son maximum $\Delta n\left(x_{\max }\right)_{t}$ est d'autant plus grand que les temps $t$ considérés sont petits devant la durée de vie $\tau_{\mathrm{B}}$ des porteurs à l'intérieur du matériau, c'est-à-dire que les porteurs injectés ont peu diffusés ou se sont peu recombinés dans le volume de l'échantillon. Comme discuté dans l'analyse du paragraphe 3 suivant, l'estimation expérimentale de $S$ sera donc facilitée par l'étude de l'excès de porteurs pour des temps brefs ( $t$ de l'ordre de $\delta$ ) dans le cas d'une excitation par lumière suffisamment absorbée.

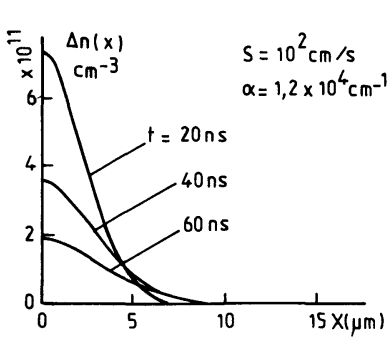

a1

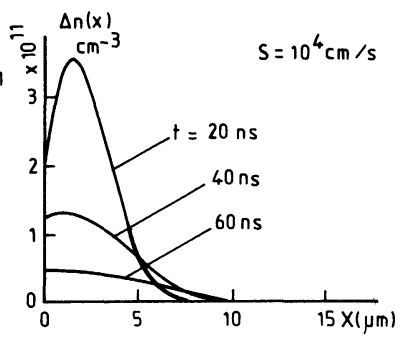

a2

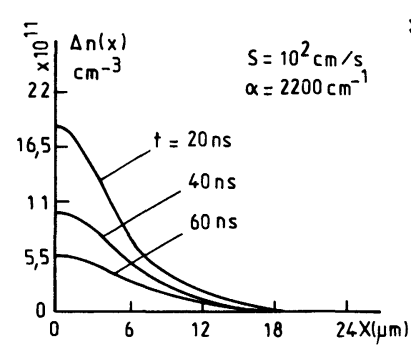

b1 b2

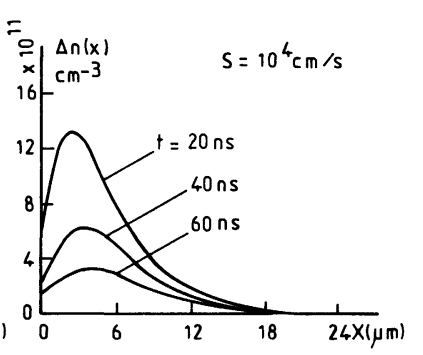

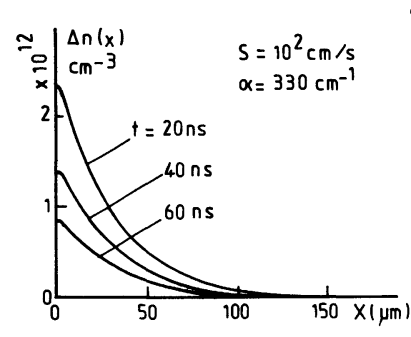

cl

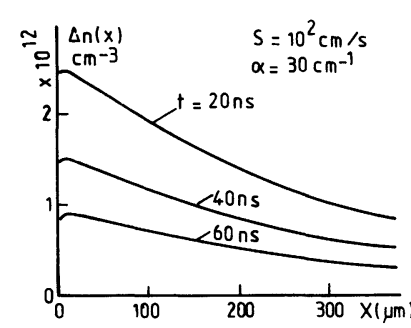

d1

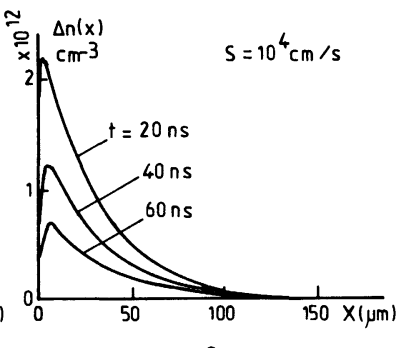

c2

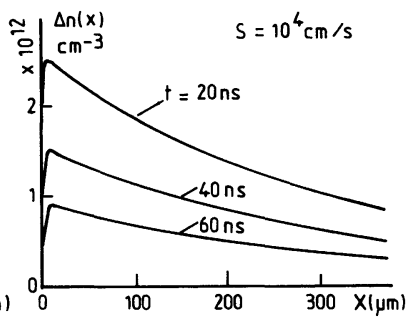

d2
Fig. 1. - Effet de la vitesse de recombinaison de surface $\left(S=10^{2}\right.$ ou $\left.10^{4} \mathrm{~cm} / \mathrm{s}\right)$ sur le profil de porteurs $\Delta n(x)_{t}$ dans un échantillon semi-infini.

Cas d'un film de silicium ( $\left.D=1 \mathrm{~cm}^{2} / \mathrm{s}, \tau_{\mathrm{B}}=40 \mathrm{~ns}\right)$ soumis à des impulsions lumineuses rectangulaires de durée $\delta=$ $0,4 \mathrm{~ns}$ et de différent coefficient d'absorption $\alpha$ :
1. a 1,2
$\alpha=1,2 \times 10^{4} \mathrm{~cm}^{-1}$
1. b $1,2 \quad \alpha=2,2 \times 10^{3} \mathrm{~cm}^{-1}$
1. c $1,2 \quad \alpha=330 \mathrm{~cm}^{-1}$
1. d $1,2 \alpha=30 \mathrm{~cm}^{-1}$.

[Changes with the surface recombination velocity $S=10^{2}$, $10^{4} \mathrm{~cm} / \mathrm{s}$ in the calculated excess carrier distribution $v s$ depth $\Delta n(x)_{t}$ in a semi-infinite sample.

Silicon layer $\left(D=1 \mathrm{~cm}^{2} / \mathrm{s}, \tau_{\mathrm{B}}=40 \mathrm{~ns}\right)$ illuminated by rectangular pulses of $\delta=0.4$ ns duration and various absorption coefficient $\alpha$ :
1. a 1,2
$\alpha=1.2 \times 10^{4} \mathrm{~cm}^{-1}$
1. b 1,2
$\alpha=2.2 \times 10^{3} \mathrm{~cm}^{-1}$
1. c 1,2
$\alpha=330 \mathrm{~cm}^{-1}$
1. d 1,2
$\alpha=30 \mathrm{~cm}^{-1}$.] 

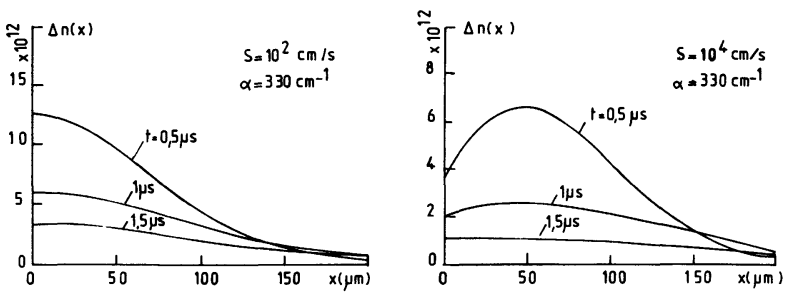

$a_{1}$

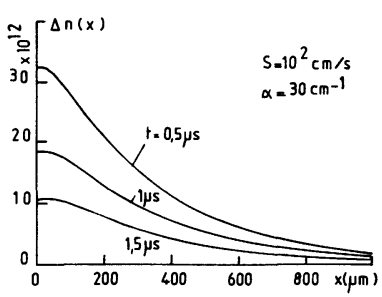

$\mathrm{b}_{1}$

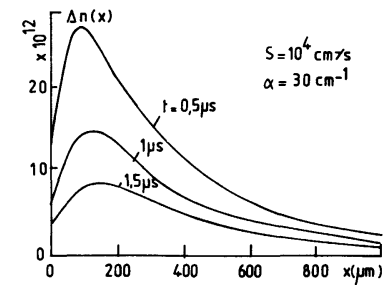

$b_{2}$

Fig. 2. - Effet de la vitesse de recombinaison de surface $\left(S=10^{2}\right.$ ou $\left.10^{4} \mathrm{~cm} / \mathrm{s}\right)$ sur le profil de porteurs $\Delta n(x)_{t}$ dans un échantillon semi-infini.

Cas d'un substrat de silicium $\left(D=34 \mathrm{~cm}^{2} / \mathrm{s}, \tau_{\mathrm{B}}=1 \mu \mathrm{s}\right)$ soumis à des impulsions lumineuses rectangulaires de durée $\delta=60$ ns et de coefficient d'absorption :

2. a $1,2 \quad \alpha=330 \mathrm{~cm}^{-1}$

2. b $1,2 \quad \alpha=30 \mathrm{~cm}^{-1}$.

[Changes with the surface recombination velocity $S=10^{2}$, $10^{4} \mathrm{~cm} / \mathrm{s}$ in the calculated excess carrier distribution $v s$. depth $\Delta n(x)_{t}$ in a semi-infinite sample.

Silicon substrate $\left(D=34 \mathrm{~cm}^{2} / \mathrm{s}, \tau_{\mathrm{B}}=1 \mu \mathrm{s}\right)$ illuminated by rectangular pulses of $\delta=60 \mathrm{~ns}$ duration and absorption coefficient :

$\begin{array}{ll}\text { 2. a } 1,2 & \alpha=330 \mathrm{~cm}^{-1} \\ \text { 2. b } 1,2 & \alpha=30 \mathrm{~cm}^{-1} \text {.] }\end{array}$

2) La deuxième remarque concerne l'influence de l'épaisseur finie d'un échantillon réel : un échantillon peut être considéré comme infini lorsque, pour le domaine de temps $t$ concerné, les porteurs injectés n'atteignent pas sa limite arrière. C'est le cas des excitations par lumières relativement absorbées, telles que $1 / \alpha \ll W$, avec des temps suffisamment brefs pour que soit satisfaite la condition $1 / \alpha+\sqrt{D t} \ll W$. Pour les matériaux et conditions d'excitations traités sur les figures 1 et 2 , cette condition reste par exemple vérifiée sur un très large domaine de temps avec un substrat de silicium d'épaisseur $W \geqslant 200 \mu \mathrm{m}$ si $\alpha=$ $330 \mathrm{~cm}^{-1}(\lambda=0,91 \mu \mathrm{m})$, ou avec un film d'épaisseur $W \geqslant 10 \mu \mathrm{m}$ si $\alpha=1,2 \times 10^{4}(=0,51 \mu \mathrm{m})$. La vitesse de recombinaison en surface $S_{x=0}$ et la durée de vie volumique $\tau_{\mathrm{B}}$ sont alors les deux paramètres (associés au matériau) qui déterminent la distribution $\Delta n(x, t)$ de porteurs.

Par contre, lorsque la condition $1 / \alpha+\sqrt{D t} \ll W$ n'est pas satisfaite, la dimension finie de l'échantillon doit être prise en compte : l'expression (3) n'est plus valable puisqu'elle ne tient pas compte des pertes à la face arrière $x=W$. L'excès de porteurs ne se réduit alors à une expression simple des paramètres $S$ et $\tau_{\mathbf{B}}$ qui caractérisent l'échantillon que si l'injection peut être considérée comme uniforme sur son épaisseur $W$. Nous avons donné sur la figure 3, en fonction du temps $t$ et pour les conditions traitées sur les figures 1 et 2 ,
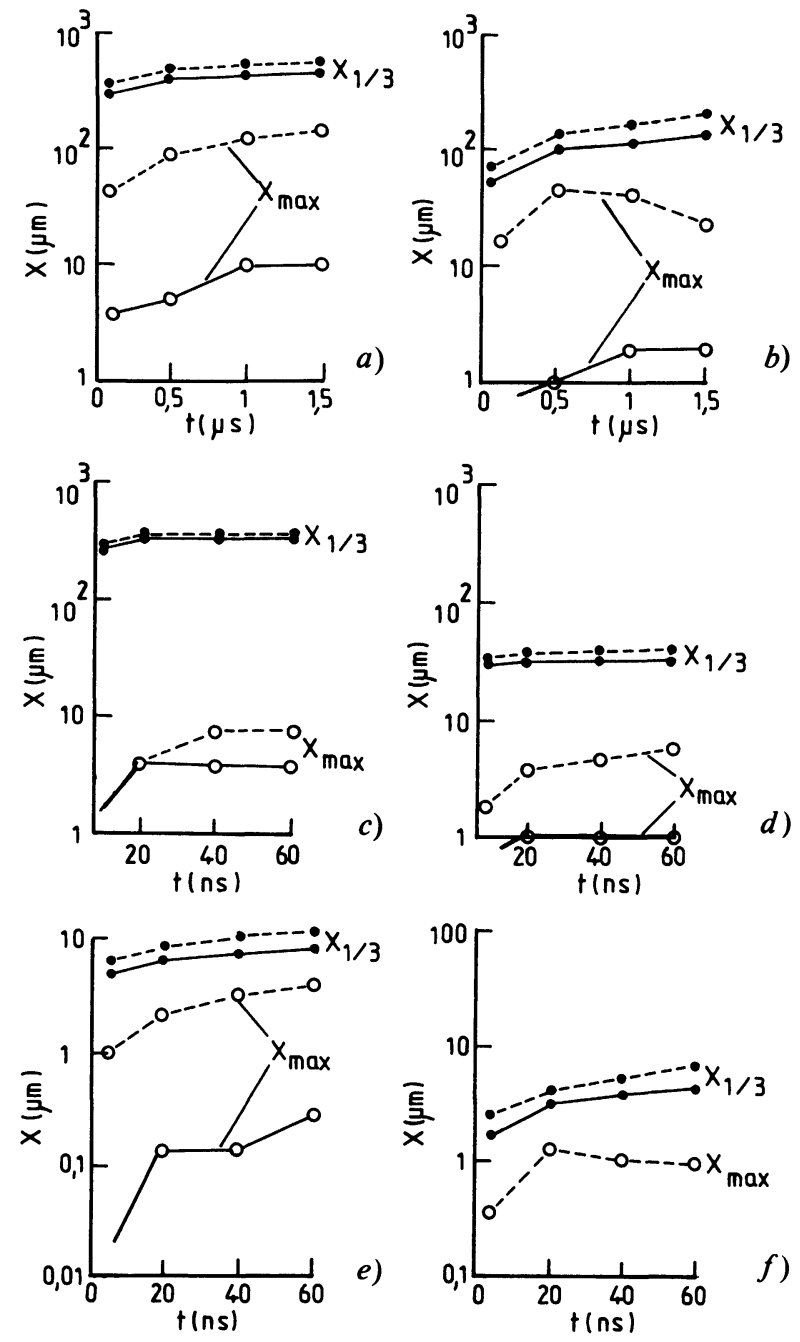

Fig. 3. - Evolution avec le temps $t$ des profondeurs $x_{\max }$ et $x_{1 / 3}$ auxquelles l'excès $\Delta n$ de porteurs est respectivement maximum $\Delta n_{\max }$ et égal à $\Delta n_{\max } / 3$, pour $S=10^{2}$ (一) et $S=10^{4}(---) \mathrm{cm} / \mathrm{s}$.

3. a, b : Cas d'un substrat ( $\left.D=34 \mathrm{~cm}^{2} / \mathrm{s}, \tau_{\mathrm{B}}=1 \mu \mathrm{s}\right)$ soumis à une impulsion lumineuse rectangulaire $\delta=60 \mathrm{~ns}: \alpha=30$ et $330 \mathrm{~cm}^{-1}$.

3. c, d, e, f : Cas d'un film ( $\left.D=1 \mathrm{~cm}^{2} / \mathrm{s}, \tau_{\mathrm{B}}=40 \mathrm{~ns}\right)$ soumis à une impulsion lumineuse rectangulaire $\delta=4 \mathrm{~ns}: \alpha=30$, $330,2,2 \times 10^{3}$ et $1,2 \times 10^{4} \mathrm{~cm}^{-1}$.

[Variation with time $t$ of the sample depths $x_{\max }$ and $x_{1 / 3}$ corresponding to the maximum $\Delta n_{\max }$ and $\Delta n_{\max } / 3$ values of the excess carrier distribution, respectively.

Cases $S=10^{2}(-)$ and $S=10^{4}(---) \mathrm{cm} / \mathrm{s}$.

3. a, b : Silicon substrate $\left(D=34 \mathrm{~cm}^{2} / \mathrm{s}, \tau_{\mathbf{B}}=1 \mu \mathrm{s}\right)$ illuminated by rectangular pulses of $\delta=60$ ns duration : $\alpha=30$ and $330 \mathrm{~cm}^{-1}$.

3. c, d, e, f : Silicon layer $\left(D=1 \mathrm{~cm}^{2} / \mathrm{s}, \tau_{\mathrm{B}}=40 \mathrm{~ns}\right)$ submitted to rectangular light pulses $\delta=40 \mathrm{~ns}: \alpha=30,330$, $2.2 \times 10^{3}$ and $1.2 \times 10^{4} \mathrm{~cm}^{-1}$.] 
à la fois les valeurs de $x_{\max }$ et celles de $x_{1 / 3}$ telles que $\Delta n\left(x_{1 / 3}\right)=\Delta n\left(x_{\max }\right) / 3$. Comme $x_{1 / 3}$ est toujours grand devant $x_{\max }$, on peut en pratique considérer que les valeurs de $x_{1 / 3}$ données dans la figure 3 correspondent à l'épaisseur maximale $W_{\max }$ que peut avoir un échantillon pour que l'injection de porteurs puisse y être considérée quasi-uniforme. Sous ces conditions, la décroissance temporelle de l'excès de porteurs sera décrite par une durée de vie effective $\tau$, fonction de $\tau_{\mathbf{B}}$ et des pertes aux surfaces $S_{x=0, w}$ :

$$
\tau^{-1}=\tau_{\mathrm{B}}^{-1}+\tau_{\mathrm{G}}^{-1}\left(S_{0}, S_{w}, D, W\right)
$$

(i) Si les vitesses de recombinaison aux surfaces sont égales, $S_{0}=S_{w}=S, \tau_{\mathrm{G}}$ prend l'expression [5],

$$
\tau_{\mathrm{G}}(S)=\frac{W^{2}}{\eta^{2} \pi^{2} D}
$$

avec $\eta$ solution de l'équation

$$
\operatorname{cotan}(\eta \pi / 2)-\eta \pi / S W=0
$$

ce qui donne $\tau_{\mathrm{G}}=W / 2 S$ et $W^{2} / \pi^{2} D$ lorsque $S$ tend respectivement vers zéro et l'infini.

(ii) Si $S_{0}$ et $S_{w}$ diffèrent, les expressions de $\tau_{G}$ restent simples si l'une de ces vitesses de recombinaison $\left(S_{1}\right)$ peut être considérée comme nulle ou infinie [6] :

$$
\tau_{\mathrm{G}}\left(0, \infty ; S_{2}\right)=1 / \xi_{0, \infty}^{2} D
$$

avec $\xi_{0, \infty}$ solutions de

$$
\xi_{0} \tan \left(\xi_{0} W\right)=S_{2} / D \text { et } \xi_{\infty} \operatorname{cotan}\left(\xi_{\infty} \mathrm{W}\right)=
$$

ce qui donne, lorsque $S_{2}$ croît lui-même de 0 à l'infini. $\tau_{\mathrm{G} 0}$ décroissant de quasiment l'infini à la valeur $4 W^{2} / \pi^{2} D$, et $\tau_{G \infty}$ décroissant de $4 W^{2} / \pi^{2} D$ à la valeur $W^{2} / \pi^{2} D$.

3) La troisième remarque relève de l'hypothèse d'homogénéité électrique de l'échantillon. En particulier pour un film, ceci suppose qu'il n'y a pas d'effet spécifique associé à la courbure de bande au voisinage de la surface. L'expression de base (3) implique en effet que l'injection de porteurs reste du domaine des faibles injections $\Delta n(x) \ll\left(n_{0}+p_{0}\right)$ et que cette courbure, et les états associés de surface, sont peu affectés par l'excitation lumineuse. L'analyse menée n'est par suite valable que si la profondeur $x_{0} \sim$ $N_{S S} /\left(n_{0}, p_{0}\right)$ de la courbure reste négligeable à l'échelle des conditions expérimentales $1 / \alpha$ et $W$ mises en jeu : la vitesse de recombinaison $S$ inclut alors l'effet de cette courbure vis-à-vis de l'excès $\Delta n(x, t)$ de porteurs.

\section{Effets relatifs de $S, \tau_{\mathrm{B}}, \delta$ et $\alpha$ sur l'excès intégré de porteurs (impulsion excitatrice rectangulaire).}

\subsection{EXPRESSION ANALYTIQUE ET CALCUL NUMÉRIQUE} DE $\Delta \mathcal{N}(t)$. - Le nombre total de porteurs en excès créés, par unité de surface, dans l'échantillon est donné par l'intégrale :

$$
\Delta \mathcal{N}(t)=\int_{0}^{\infty} \Delta n(x, t) \mathrm{d} x
$$

qui peut s'écrire, pour une impulsion rectangulaire de durée $\delta$ [2], sous la forme explicite suivante :

$$
\begin{aligned}
& \Delta \mathcal{N}^{*}(t)=\frac{g_{0} \tau_{\mathrm{B}} L \mathrm{e}^{-t / \tau_{\mathbf{B}}}}{\left(\alpha^{2} L^{2}-1\right)\left[(S L / D)^{2}-1\right][(S L / D)-\alpha L]} \cdot\left\{\left[\alpha^{2} L^{2}-\left(\frac{S L}{D}\right)^{2}\right] \times\right.(S L / D) \mathrm{e}^{t / \tau_{\mathbf{B}}} \cdot \operatorname{erfc}\left(\sqrt{t / \tau_{\mathbf{B}}}\right)+\left[(S L / D)^{2}-1\right] \cdot S /(\alpha D) \cdot \mathrm{e}^{\alpha^{2} L 2 t / \tau_{\mathbf{B}}} \\
&\left.\operatorname{erfc}\left(\alpha L \sqrt{t / \tau_{\mathbf{B}}}\right)-\left(\alpha^{2} L^{2}-1\right) \cdot \mathrm{e}^{(S L / D)^{2} t / \tau_{\mathbf{B}}} \operatorname{erfc}\left(\frac{S L}{D} \sqrt{t / \tau_{\mathbf{B}}}\right)\right\} \\
&+\frac{g_{0} \tau_{\mathbf{B}} / \alpha}{\left(\alpha^{2} L^{2}-1\right)} \cdot\left\{-1+L^{2} \frac{(1+S / \alpha D)}{(1+S L / D)}\right\} .
\end{aligned}
$$

avec :

$$
\begin{aligned}
& \Delta \mathcal{N}(t)=\Delta \mathcal{N}^{*}(t) \text { pour } t<\delta \\
& \Delta \mathcal{N}(t)=\Delta \mathcal{N}^{*}(t)-\Delta \mathcal{N}^{*}(t-\delta) \text { pour } t \geqslant \delta
\end{aligned}
$$

Comme illustré par les exemples des figures 4 et 5 , à la fois la croissance pour $t<\delta$ et la décroissance $t>\delta$ de $\Delta \mathcal{N}(t)$, et son amplitude à $t=\delta$, varient avec les valeurs de la vitesse de recombinaison de surface $S$ et de la durée de vie volumique $\tau_{B}$ dans l'échantillon. Ces variations sont alors d'autant plus fortes que, à valeur fixe de $D$ : (i) pour une lumière de pénétration $1 / \alpha$ donnée, le rapport $\delta / \tau_{\mathbf{B}}$ entre durée du flash et durée de vie volumique est petit, ou (ii) à $\delta / \tau_{\mathrm{B}}$ donné, la pénétration $1 / \alpha$ est plus faible.

3.2 Utilisation DE LA CROISSANCE DE $\Delta \mathcal{N}(t)$ À L'Évaluation DE $S$. - A partir des illustrations cidessus, il apparaît donc que les variations de l'allure de $\Delta \mathcal{N}(t)$ avec $S, \tau_{\mathrm{B}}, \alpha$ ou $\delta$ correspondent à des variations non seulement de la décroissance mais aussi du rapport

$$
K=\Delta \mathcal{N}^{\prime}\left(\delta_{+}\right) / \Delta \mathcal{N}^{\prime}\left(\delta_{-}\right)
$$



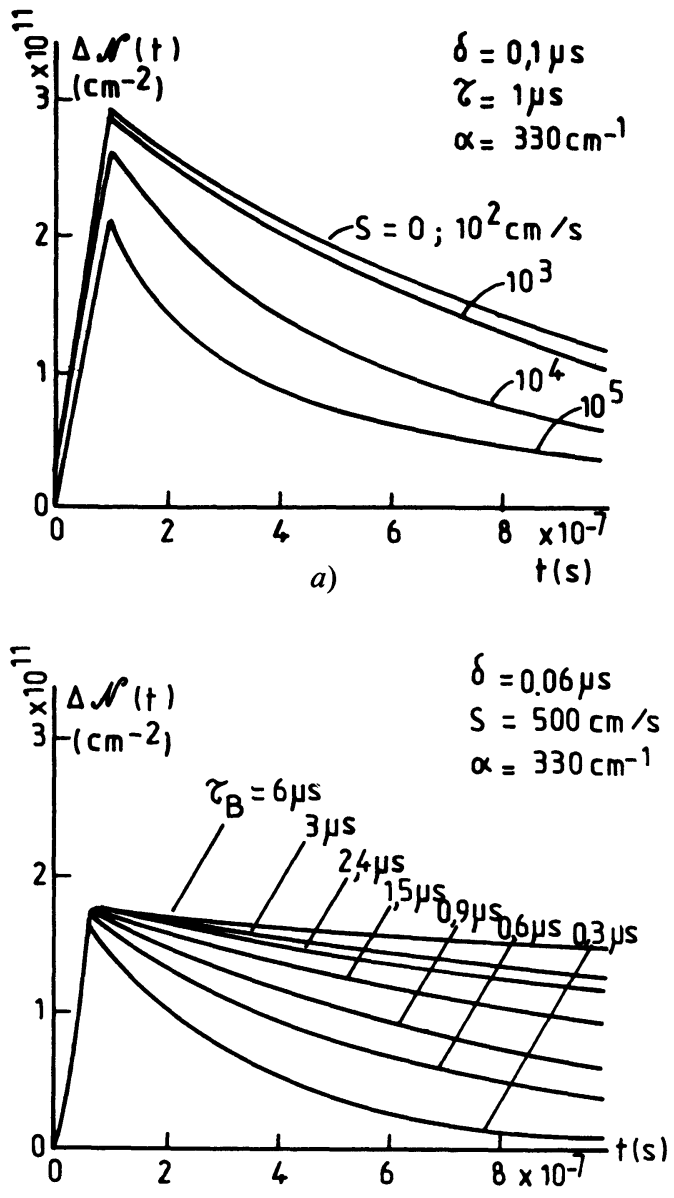

b)

Fig. 4. - Effets de la vitesse de recombinaison de surface $S$ et de la durée de vie de volume $\tau_{\mathrm{B}}$ sur l'évolution avec le temps $t$ de l'excès intégré de porteurs $\Delta \mathcal{N}(t)$ créé dans un échantillon semi-infini.

Cas d'un substrat de silicium $\left(D=34 \mathrm{~cm}^{2} / \mathrm{s}\right)$ soumis à une impulsion rectangulaire de diode laser à l'AsGa $(\alpha=$ $\left.330 \mathrm{~cm}^{-1}\right)$ :

4. a : $\tau_{\mathrm{B}}=1 \mu \mathrm{s}, S=0$ à $10^{5} \mathrm{~cm} / \mathrm{s}(\delta=100 \mathrm{~ns})$

4. b : $S=5 \times 10^{2} \mathrm{~cm} / \mathrm{s}, \tau_{\mathrm{B}}=0,3$ à $6 \mu \mathrm{s}(\delta=60 \mathrm{~ns})$.

[Changes in the integrated excess carrier variation $v s$. time $\Delta \mathcal{N}(t)$ with surface recombination velocity $S$ and bulk lifetime $\tau_{\mathbf{B}}$ of a semi-infinite sample. Silicon substrate illuminated by a rectangular GaAs laser pulse $(\alpha=$ $330 \mathrm{~cm}^{-1}$ ) :

4. a : $\tau_{\mathrm{B}}=1 \mu \mathrm{s}, S=0$ to $10^{5} \mathrm{~cm} / \mathrm{s}(\delta=100 \mathrm{~ns})$

4. b : $S=5 \times 10^{2} \mathrm{~cm} / \mathrm{s}, \tau_{\mathrm{B}}=0.3$ to $6 \mu \mathrm{s}(\delta=60 \mathrm{~ns})$.]

entre les dérivées de $\Delta \mathcal{N}(t)$ de part et d'autre du temps $t=\delta$ auquel cesse l'excitation lumineuse.

3.2.1 Estimation numérique du rapport $K\left(S, \tau_{\mathrm{B}}, D\right)$ pour $\alpha$ et $\delta$ donnés. - La figure 6 , par exemple, donne les valeurs $K_{v}(S)$ obtenues, en fonction de $\tau_{\mathrm{B}}$ et pour $D$, $\alpha$ et $\delta$ donnés, par dérivation numérique de l'évolution de l'excès total $\Delta \mathcal{N}(t)$ calculé par l'expression(13), soit :

$$
K_{v}(S)=\frac{\Delta \mathcal{N}(t=\delta)-\Delta \mathcal{N}(t=\delta+\varepsilon)}{\Delta \mathcal{N}(t=\delta-\varepsilon)-\Delta \mathcal{N}(t=\delta)} .
$$
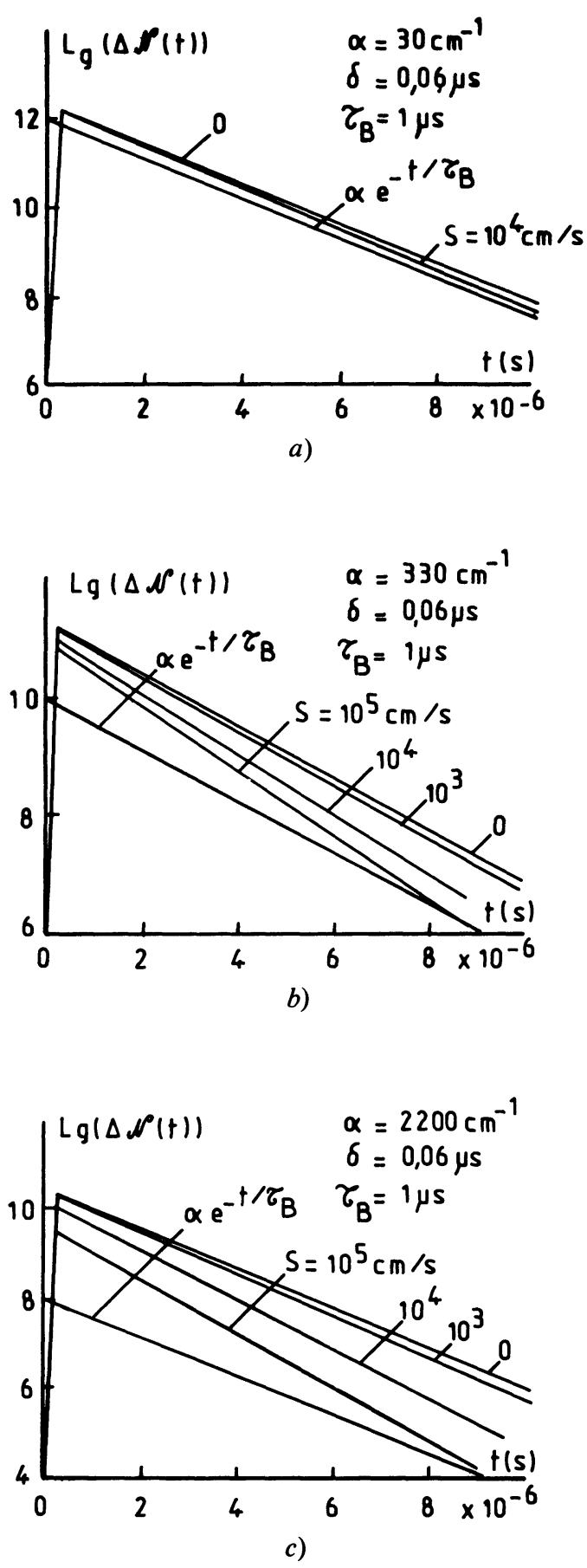

Fig. 5. - Effet du coefficient d'absorption $\alpha$ de la longueur d'onde excitatrice sur l'excès intégré de porteurs $\Delta \mathcal{N}(t)$. Cas d'un substrat de silicium $D=34 \mathrm{~cm}^{2} / \mathrm{s}, \tau_{\mathrm{B}}=1 \mu \mathrm{s}$, $S=0$ à $10^{5} \mathrm{~cm} / \mathrm{s}$, soumis à des impulsions rectangulaires de durées $\delta=60 \mathrm{~ns}$ :

5. a $\alpha=30 \mathrm{~cm}^{-1}$

5. b $\quad \alpha=330 \mathrm{~cm}^{-1}$

5. c $\alpha=2,2 \times 10^{3} \mathrm{~cm}^{-1}$.

[Changes in the integrated excess carrier density $\Delta \mathcal{N}(t)$ with absorption coefficient $\alpha$ of the excitating light.

Silicon substrate $D=34 \mathrm{~cm}^{2} / \mathrm{s}, \tau_{\mathrm{B}}=1 \mu \mathrm{s}, S=0$ to $10^{5} \mathrm{~cm} / \mathrm{s}$ illuminated by rectangular pulses of $\delta=60$ ns duration :

5. a $\alpha=30 \mathrm{~cm}^{-1}$

5. b $\quad \alpha=330 \mathrm{~cm}^{-1}$

5. c $\alpha=2.2 \times 10^{3} \mathrm{~cm}^{-1}$.] 


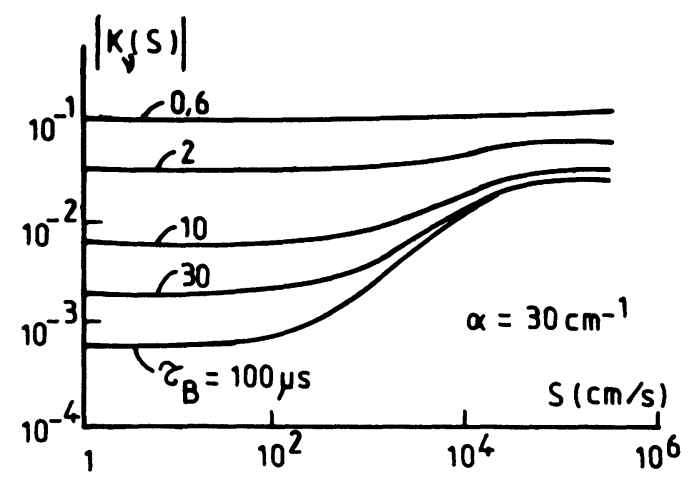

a)

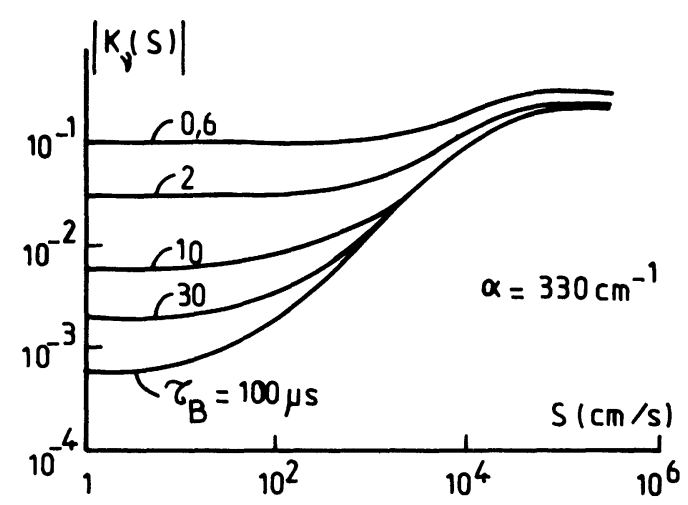

b)

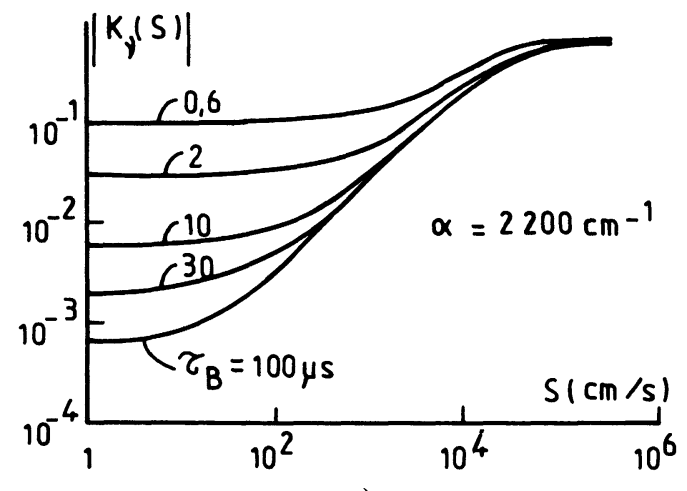

c)

Fig. 6. - Effet de la durée de vie de volume $\tau_{\mathbf{B}}$ sur la variation avec la vitesse de recombinaison en surface $S$ du rapport négatif de pentes $K_{v}$ calculé par dérivation numérique de l'expression (13) de l'excès de porteurs $\Delta \mathcal{N}(t)$.

Cas d'un substrat de silicium $\left(D=34 \mathrm{~cm}^{2} / \mathrm{s}, \tau_{\mathrm{B}}=0,6\right.$ à $100 \mu \mathrm{s})$ éclairé par des impulsions lumineuses rectangulaires de durée $\delta=60 \mathrm{~ns}$ avec :
6. a $\alpha=30 \mathrm{~cm}^{-1}$
6. b $\alpha=330 \mathrm{~cm}^{-1}$
6. c $\alpha=2,2 \times 10^{3} \mathrm{~cm}^{-1}$.

[Changes with bulk lifetime $\tau_{\mathrm{B}}$ of the negative ratio $K_{v}$, of the slopes of $\Delta \mathcal{N}(t)$, at $t=\delta \pm \varepsilon$, as a function of the surface recombination velocity $S$. The $K_{v}(S)$ values are obtained by numerical derivation of $\Delta \mathcal{N}(t)$ as given by expression (13).

Silicon substrate $\left(D=34 \mathrm{~cm}^{2} / \mathrm{s}, \tau_{\mathbf{B}}=0.6\right.$ à $\left.100 \mu \mathrm{s}\right)$ illuminated by rectangular light pulses of $\delta=60$ ns duration :
6. a $\alpha=30 \mathrm{~cm}^{-1}$
6. b $\quad \alpha=330 \mathrm{~cm}^{-1}$
6. c $\alpha=2.2 \times 10^{3} \mathrm{~cm}^{-1}$.]

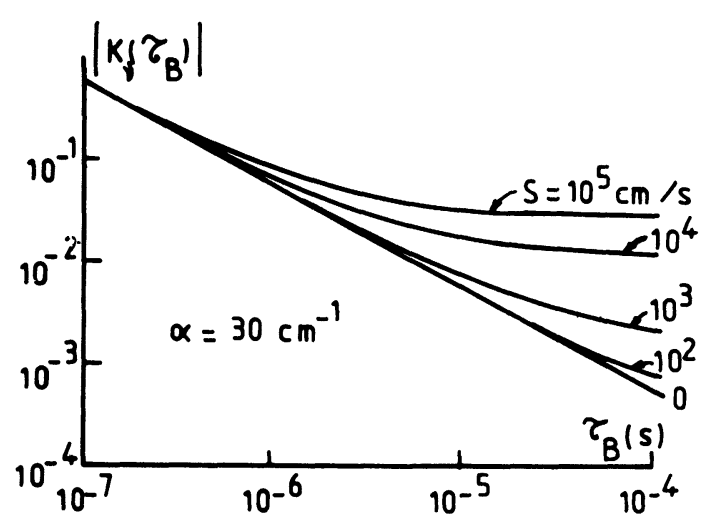

a)

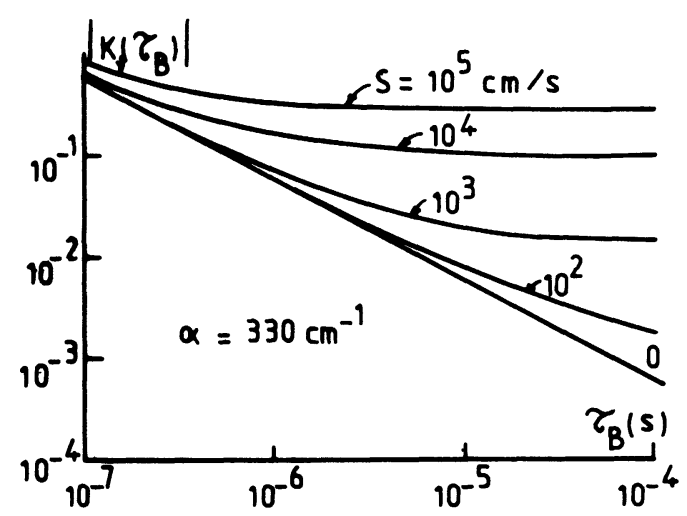

b)

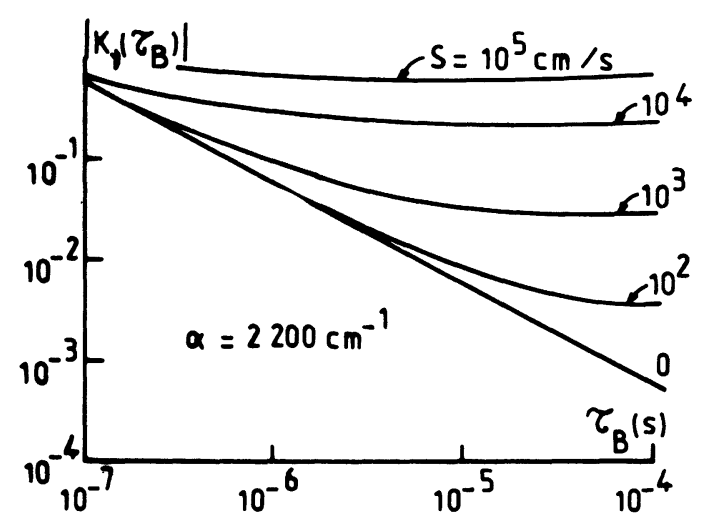

c)

Fig. 7. - Effet de la valeur de $S$ sur l'évolution avec $\tau_{\mathbf{B}}$ du rapport négatif de pente $K_{v}\left(\tau_{\mathrm{B}}\right)$ calculé par dérivation numérique de (13).

Cas d'un substrat de silicium $\left(D=34 \mathrm{~cm}^{2} / \mathrm{s}, S=0\right.$ à $10^{5} \mathrm{~cm} / \mathrm{s}$ ) et d'impulsions lumineuses rectangulaires de durée $\delta=60 \mathrm{~ns}$ avec :
7. a $\alpha=30 \mathrm{~cm}^{-1}$
7. b $\quad \alpha=330 \mathrm{~cm}^{-1}$
7. c $\alpha=2,2 \times 10^{3} \mathrm{~cm}^{-1}$.

[Changes with surface recombination velocity $S$ of the negative ratio $K_{v}$ as a function of bulk lifetime $\tau_{\mathrm{B}}$. Silicon substrate $\left(D=34 \mathrm{~cm}^{2} / \mathrm{s}, S=0\right.$ to $\left.10^{5} \mathrm{~cm} / \mathrm{s}\right)$; rectangular light pulses of $\delta=60 \mathrm{~ns}$ duration with :
7. a $\alpha=30 \mathrm{~cm}^{-1}$
7. b $\alpha=330 \mathrm{~cm}^{-1}$
7. c $\alpha=2.2 \times 10^{3} \mathrm{~cm}^{-1}$.] 
Dans la figure 6 qui traite de l'exemple d'un substrat de silicium $\left(D=34 \mathrm{~cm}^{2} / \mathrm{s}, \tau_{\mathrm{B}}=0,6\right.$ à $\left.100 \mu \mathrm{s}\right)$ on voit ainsi que, pour $\tau_{\mathbf{B}}$ donné et dès que $S$ cesse d'être nul ou très faible $(S>10 \mathrm{~cm} / \mathrm{s})$, l'amplitude du rapport de pentes $K$ est d'autant plus grande que la lumière est plus fortement absorbée.

La figure 7 reprend ces mêmes résultats en donnant l'évolution correspondante $K_{v}\left(\tau_{B}\right)$ pour différentes valeurs de $S$, ce qui peut faciliter l'exploitation expérimentale de tels résultats.
3.2.2 Traitement analytique. - L'intérêt du traitement analytique est de permettre l'évaluation quantitative du rapport de pentes $K$ en fonction des différents paramètres $\alpha, \delta$ ou $S, \tau_{\mathrm{B}}$, mais aussi $D$, mis en jeu dans l'étude. A ce titre, les deux expressions (17) et (18) de $K$, discutées ci-après, peuvent être utilisées. Sur le plan analytique, il suffit en effet que les temps $t=\delta \pm \varepsilon$ considérés, et donc la durée $\delta$ du flash d'excitation, soient suffisamment courts pour que le rapport $K$ puisse être décrit et calculé par l'expression :

$$
K_{\mathrm{a} 1}=\frac{S / \alpha \cdot D-1}{(S / \alpha D) \operatorname{Exp}\left(\left(\alpha^{2} L^{2}-1\right) S / \tau_{\mathrm{B}}\right)-\operatorname{Exp}\left(\left(\left(\frac{S L}{D}\right)^{2}-1\right) \delta / \tau_{\mathrm{B}}\right)}-1
$$

où $\delta$ doit être assez petit pour que soient satisfaites les conditions :

$$
\sqrt{\delta / \tau_{\mathrm{B}}}, \quad S \sqrt{\delta / D}, \quad \text { et } \quad \alpha \sqrt{D \delta} \ll 1 .
$$

$\mathrm{Si}$, de plus, la vitesse de recombinaison en surface $S$ est suffisamment faible, l'expression (17) précédente se réduit à la forme simple et pratique suivante :

$$
K_{\mathrm{a} 2}=\alpha \delta S+\delta / \tau_{\mathrm{B}}
$$

pour des temps très courts (cf. (16)) avec

$$
S \ll \alpha D \text { et } S \ll \sqrt{D / \tau_{\mathbf{B}}} .
$$

On peut remarquer que l'expression simplifiée (18) implique donc que la vitesse de recombinaison en surface $S$ est très petite devant à la fois ce qu'on peut appeler une "vitesse de génération » $\alpha D$, et la vitesse de diffusion en volume $L / \tau_{B}=\sqrt{D / \tau_{B}}$, des porteurs.

(Dans la pratique, l'intérêt de cette expression simplifiée (18) est d'aider à situer, pour un échantillon donné, les valeurs théoriques du rapport de pentes $K_{\mathrm{a}}\left(S, \tau_{\mathrm{B}}\right)$ qui correspondent aux paramètres $\alpha, \delta$ utilisés. La figure 8 donne les valeurs $K_{\mathrm{a} 2}$ calculées par (18) pour les trois cas traités numériquement sur la figure 6. La comparaison entre les deux groupes de valeurs $K_{\mathrm{a} 2}$ et $K_{v}$ du rapport de pentes obtenues

Fig. 8. - Rapport négatif de pentes $K_{\mathrm{a}}(S)$ donné par l'expression simplifiée (18) pour les cas considérés sur la figure 7 . Substrat de silicium $D=34 \mathrm{~cm}^{2} / \mathrm{s}$ et impulsions lumineuses rectangulaires $\delta=10$ ns avec (cf. Fig. 8) :
8. a $\alpha=30 \mathrm{~cm}^{-1}$
8. b $\alpha=330 \mathrm{~cm}^{-1}$
8. c $\alpha=2 \times 10^{3} \mathrm{~cm}^{-1}$.

[Negative slope ratio $K_{\mathrm{a}}(S)$ calculated as a function of $S$ using the simple form (18). Silicon substrate and rectangular $60 \mathrm{~ns}$ pulse (cf. Fig. 8) :

8. a $\alpha=30 \mathrm{~cm}^{-1}$

8. b $\quad \alpha=330 \mathrm{~cm}^{-1}$

8. c $\alpha=2.2 \times 10^{3} \mathrm{~cm}^{-1}$.]

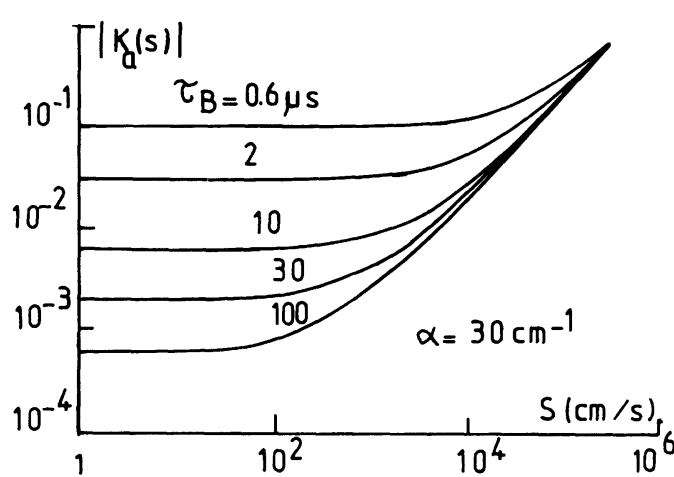

a)

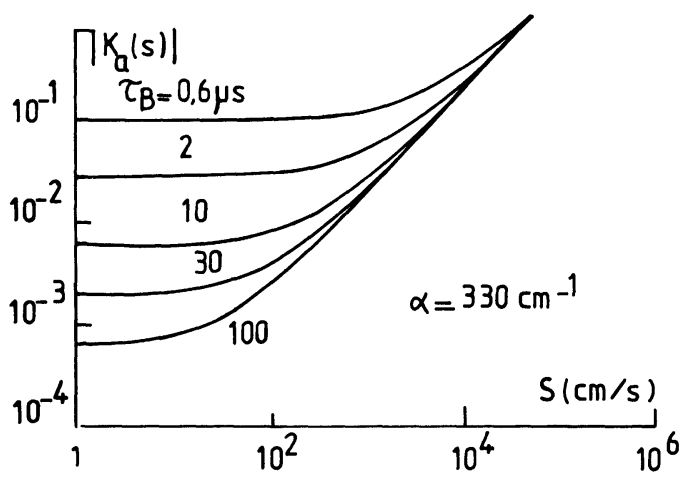

b)

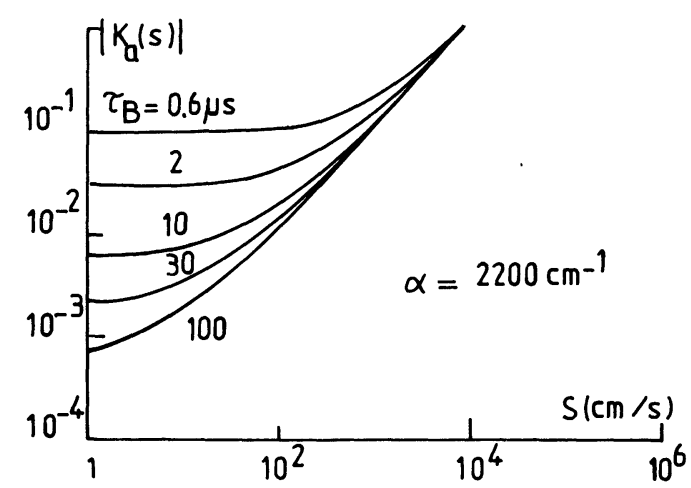

c) 
d'une part à partir de la relation simplifiée (18) (cf. Fig. 8), et d'autre part à partir du calcul numérique de l'expression de base (cf. Fig. 6) est traitée sur la figure 9. L'étude illustre l'effet de $S$ sur l'erreur relative $\left(K_{\mathrm{a} 2}-K_{v}\right) / K_{v}$ faite dans l'utilisation de (18).)
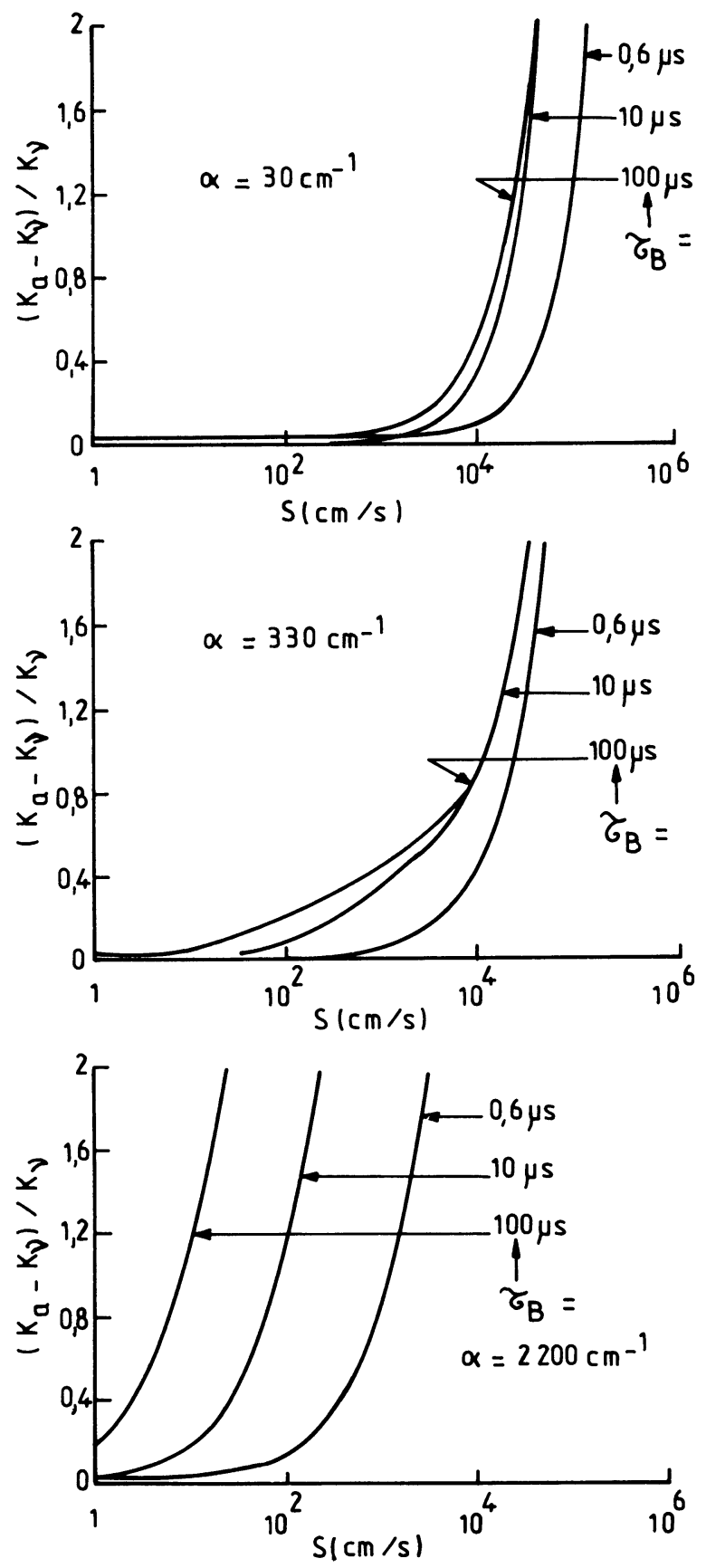

Fig. 9. - Variation de l'erreur relative $\left(K_{\mathrm{a}}-K_{\mathrm{v}}\right) / K_{\mathrm{v}}$ avec la vitesse de recombinaison en surface $S$ pour les cas considérés dans les figures 8 et 9 précédentes. (Substrat $D=$ $34 \mathrm{~cm}^{2} / \mathrm{s}, \quad \tau_{\mathrm{B}}=0,6$ à $100 \mu \mathrm{s}$; impulsions rectangulaires $\delta=60 \mathrm{~ns}$ avec $=30,330$ ou $2,210 \mathrm{~cm})$. Cas $K_{\mathrm{a}}$ donné par l'expression simplifiée (18).]

[Relative error $\left(K_{\mathrm{a}}-K_{v}\right) / K_{v}$ as a function of surface recombination velocity $S$ for the examples given in figures 8 and 9 . (Silicon substrate $D=34 \mathrm{~cm}^{2} / \mathrm{s}, \tau_{\mathrm{B}}=0.6$ à $100 \mu \mathrm{s}$; rectangular pulses $\delta=60 \mathrm{~ns}$ with $\alpha=30,330$ or $2.2 \times 10^{3} \mathrm{~cm}^{-1}$ ). $K_{\mathrm{a}}$ is calculated from its simplified expression (18).]

\section{Cas d'impulsions lumineuses d'allure gaussienne.}

Pour déterminer comment $K$ et, par suite, $S$ peuvent être atteints à partir d'une impulsion lumineuse d'allure gaussienne et de largeur à mi-hauteur $\delta$, nous avons d'abord simulé cette impulsion par un train d'impulsions créneaux. Puis, nous avons étudié à quels temps $t+$ et $t-$, situés de part et d'autre du maximum de la réponse $\Delta \mathcal{N}_{\mathrm{g}}(t)$, devraient être calculées les pentes $\Delta \mathcal{N}_{g}^{\prime}\left(2 \delta_{+}\right)$et $\Delta \mathcal{N}_{g}^{\prime}\left(2 \delta_{-}\right)$de la réponse de façon à ce que le rapport :

$$
K_{\mathrm{g}}=\frac{\Delta \mathcal{N}_{\mathrm{g}}^{\prime}\left(2 \delta_{+}\right)}{\Delta \mathcal{N}_{\mathrm{g}}^{\prime}\left(2 \delta_{-}\right)}
$$

soit identique au rapport $K(\delta \pm \varepsilon)$ (cf. $\S 3)$ qui caractérise la réponse à une impulsion rectangulaire de durée $\delta$.

Dans la figure 10 sont comparées les réponses $\Delta \mathcal{N}_{\mathrm{g}}(t)$ calculées en appliquant les expressions (3) et (14) à des séries de $C=5,11,15$ ou 19 créneaux, centrées sur l'impulsion gaussienne (Fig. 10a). Pour chacune de ces séries, la largeur des créneaux est prise égale à $2 \delta / C$ et l'amplitude est un multiple entier de l'amplitude élémentaire $A_{\mathrm{e}}$ :

$$
A_{\mathrm{e}}=2 A /(C+1)
$$

déduite de l'amplitude $A$ de l'impulsion gaussienne.

Lorsque le nombre $C$ de créneaux croît, l'aire $S_{\mathrm{c}}$ de la série de créneaux d'aire $S_{\mathrm{e} i}$ :

$$
S_{\mathrm{c}}=\sum_{i=1}^{c} S_{\mathrm{e} i}
$$

passe d'une sur-estimation à une sous-estimation de l'aire $S_{\mathbf{g}}$ de l'impulsion gaussienne :

$$
\begin{aligned}
S_{\mathrm{g}}=A \int_{0}^{\infty} \exp \left[\frac{-\left(t-t_{0}\right)^{2}}{\sigma}\right] \mathrm{d} t & =A . \sigma \times \\
\times & \frac{\sqrt{\pi}}{2}\left(1-\operatorname{erf}\left(-\frac{t_{0}}{\sigma}\right)\right)
\end{aligned}
$$

où $\sigma$ est donné par : $\sigma=\delta / 1,66$.

Les résultats de la figure 10 montrent que les trois séries de créneaux $C=11$ et surtout $C=15$ et 19 conduisent à une approche convenable de la réponse $\Delta \mathcal{N}_{\mathrm{g}}(t)$ induite par une impulsion excitatrice d'allure gaussienne. Nous avons donc utilisé dans la suite de l'étude, pour simuler la réponse $\Delta \mathcal{N}_{\mathrm{g}}(t)$ à ce type d'impulsion, la série de 15 créneaux qui correspond à la meilleure approche de l'aire $S_{\mathbf{g}}$.

Pour analyser à quel temps $t \pm \varepsilon$ doit être étudié le rapport de pentes $K_{\mathrm{g}}$ (cf. (15)), nous avons comparé les réponses $\Delta \mathcal{N}_{\mathrm{g}}(t)$ données par une impulsion lumineuse gaussienne à la réponse $\Delta \mathcal{N}(t)$ produite par une impulsion rectangulaire. Cette dernière impulsion est alors décalée de $\delta$ par rapport à l'impulsion gaussienne de façon à ce que les maxima des deux réponses $\Delta \mathcal{N}_{\mathrm{g}}(t), \Delta \mathcal{N}(t)$ coïncident (Fig. 11a). 


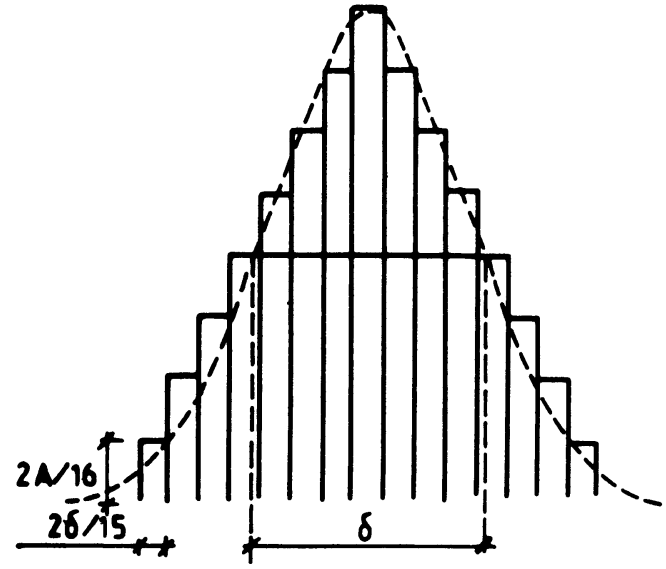

a)

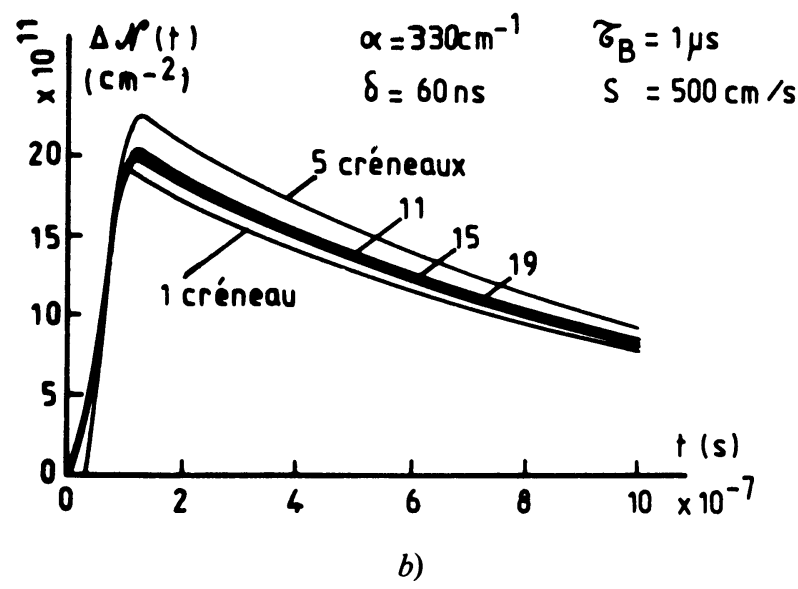

Fig. 10. - Impulsion lumineuse d'allure gaussienne et durée à mi-hauteur $\delta$ :

10. a : Schéma de la décomposition en suite d'impulsions créneaux : cas de $C=15$ impulsions, $S_{\mathrm{g}} / \mathrm{S}=64.05 \mathrm{~A} /$ 64.00 A.

10. b : Simulation de l'excès intégré de porteurs $\Delta \mathcal{N}_{\mathrm{g}}(t)$ créé par une impulsion gaussienne à partir de l'excès créé par des suites de $C=1$ à 19 créneaux.

[Gaussian shape light pulse of half-width duration $\delta$ : 10. a : Decomposition in a serie of $C=15$ rectangular pulses $\left(S_{\mathrm{g}} / S=64.05 \mathrm{~A} / 64.00 \mathrm{~A}\right)$.

10. b : Integrated carrier excess $\Delta \mathcal{N}_{\mathrm{g}}(t)$ simulated by using gaussian pulse decomposition in series of $C=1$ to 19 rectangular pulses.]

Pour ces deux types d'impulsions, nous avons effectué le calcul numérique des pentes

$$
\Delta[\Delta \mathcal{N}(t>2 \delta)] / \Delta t \text { et } \Delta[\Delta \mathcal{N}(t<2 \delta)] / \Delta t
$$

et confronté leurs évolutions dans le domaine de temps $t=0$ à $5 \delta$ qui recouvre la montée et le début de la décroissance des réponses $\Delta \mathcal{N}(t)$. Les figures $11 \mathrm{~b}$ et $11 \mathrm{c}$ illustrent les résultats obtenus pour $\alpha=$ $330 \mathrm{~cm}^{-1}$.

Cette étude montre que :

1. En ce qui concerne la décroissance $\Delta \mathcal{N}(t>2 \delta)$, la pente $\Delta\left[\Delta \mathcal{N}_{\mathrm{g}}(t>2 \delta)\right] / \Delta t$ associée à l'impulsion

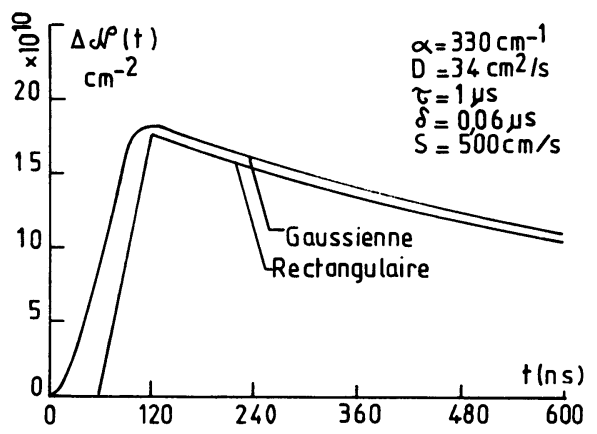

a)

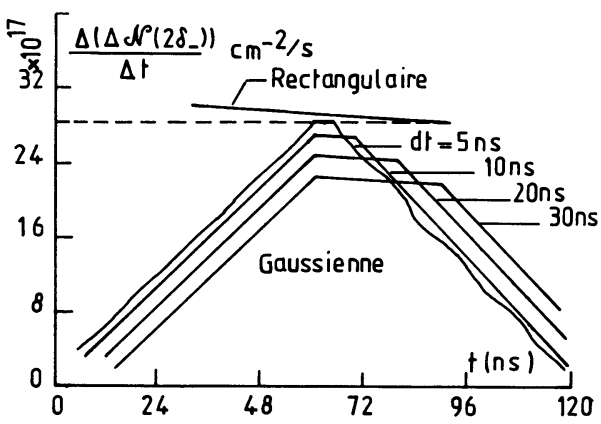

b)
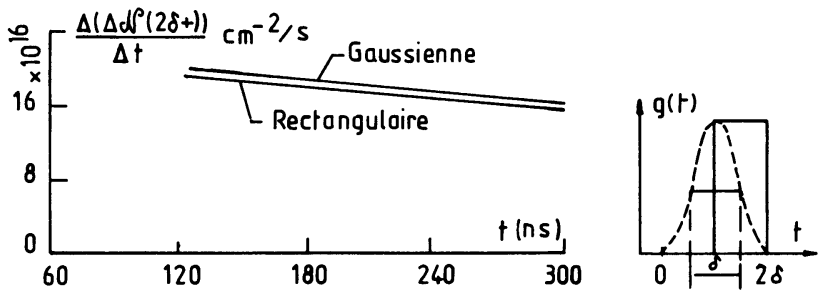

c)

Fig. 11. - Comparaison des excès intégrés de porteurs créés par une impulsion d'allure gaussienne (largeur à mihauteur $\delta$ ) et une impulsion rectangulaire de durée $\delta$, décalée de $\delta$.

Simulation de l'impulsion gaussienne par la série de $15 \mathrm{im}$ pulsions crénaux. Calculs relatifs au cas d'un substrat $\left(D=34 \mathrm{~cm}^{2} / \mathrm{s}, \tau_{\mathrm{B}}=1 \mu \mathrm{s}, S=5 \times 10^{2} \mathrm{~cm} / \mathrm{s}\right)$ éclairé par une diode laser à l'AsGa $\left(\delta=60 \mathrm{~ns}, \alpha=330 \mathrm{~cm}^{-1}\right)$.

Calcul numérique de l'évolution avec le temps $t$

11. a : de l'excès intégré de porteurs $\Delta \mathcal{N}_{\mathrm{g}}(t)$

11. b : des pentes positives $\Delta\left[\Delta \mathcal{N}_{\mathrm{g}}(t<2 \delta)\right] / \Delta t$ de la croissance de $\Delta \mathcal{N}_{\mathrm{g}}(t<2 \delta)$

11. c : des pentes négatives $\Delta\left[\mathcal{N}_{\mathrm{g}}(t>2 \delta)\right] / \Delta t$ de la décroissance de $\Delta \mathcal{N}_{\mathrm{g}}(t>2 \delta)$.

[Comparison of the integrated carrier excess induced by a Gaussian shape light pulse (half-height width $\delta$ ) and a $\delta$ delayed rectangular pulse of duration $\delta$.

The gaussian pulse is simulated by the series of $C=15$ rectangular pulses illustrated in figure 11a. Silicon substrate $\left(D=34 \mathrm{~cm}^{2} / \mathrm{s}, \tau_{\mathrm{B}}=1 \mu \mathrm{s}, S=5 \times 10^{2} \mathrm{~cm}^{2} / \mathrm{s}\right)$ illuminated by AsGa laser pulse $\delta=60 \mathrm{~ns}\left(\alpha=330 \mathrm{~cm}^{-1}\right)$.

Numerical values as a function of time $t$ of :

11. a : the integrated excess carrier density $\Delta \mathcal{N}_{\mathrm{g}}(t)$

11. b : the positive slope of the $\Delta \mathcal{N}_{\mathrm{g}}(t)$ increase

11. $\mathrm{c}$ : the negative slope of the $\Delta \mathcal{N}_{\mathrm{g}}(t)$ decay.] 
gaussienne est identique à la pente $\Delta[\Delta \mathcal{N}(t>2 \delta)] / \Delta t$ associée à l'impulsion rectangulaire sur tout l'intervalle de temps $t=2$ à $5 \delta$ considéré (cf. Fig. 11b).

2. Lors de la croissance, il n'y a correspondance entre les deux types de pentes positives que pour la valeur $\Delta\left[\Delta \mathcal{N}_{\mathrm{g}}(t=\delta)\right] / \Delta t$ considérée au point d'inflexion $t=\delta$ de la croissance de $\Delta \mathcal{N}_{\mathrm{g}}(t)$. A ce temps $t=\delta$, la valeur numérique de la pente associée à l'impulsion gaussienne est alors d'autant plus proche de celle de la pente positive associée à l'impulsion rectangulaire que l'intervalle de temps $\Delta t$ considéré autour de $t=\delta$ est petit.

Ainsi, à condition de prendre pour référence une impulsion rectangulaire décalée de $\delta$ par rapport à l'impulsion gaussienne de façon à faire coïncider les maxima des réponses $\Delta \mathcal{N}(t)$, cette étude met en relief que, lors d'une excitation par impulsion lumineuse d'allure gaussienne et de durée à mi-hauteur $\delta$ :

- la décroissance des réponses $\Delta \mathcal{N}_{\mathrm{g}}(t)$ est identique à celle que donnerait l'impulsion rectangulaire de référence, et peut donc s'exploiter de la même façon pour atteindre $\tau_{\mathbf{B}}$ ou $\tau$;

- il est également possible d'utiliser le rapport de pentes $K$ pour estimer $S$. Il faut alors mesurer la pente positive $\Delta\left[\Delta \mathcal{N}_{\mathrm{g}}(t<2 \delta)\right] / \Delta t$, relative à la croissance de $\Delta \mathcal{N}_{\mathrm{g}}(t)$, au niveau du point d'inflexion $t=\delta$ de cette croissance. La précision avec laquelle est estimée la valeur de cette pente est alors d'autant plus grande que l'intervalle de temps $\Delta t$ sur lequel est estimée la variation de $\Delta \mathcal{N}_{\mathrm{g}}(t)$ est petit. Mais cette précision (cf. Fig. 11b) reste intéressante puisqu'elle se situe entre $20 \%$ pour $\Delta t=\delta / 2$ et $5 \%$ pour $\Delta t=\delta / 6$, ce qui reste suffisant pour utiliser la mesure expérimentale de $K$ à une estimation correcte de $S$.

\section{Conclusion}

Le rapport $K$ entre les pentes mesurées de part et d'autre d'une réponse qui traduit l'excès intégré $\Delta \mathcal{N}(t)$ de porteurs créé dans un échantillon permet d'obtenir la valeur de la vitesse de recombinaison de surface, $S$, si celle de la durée de vie de volume $\tau_{\mathbf{B}}$ est connue. Ceci pour une impulsion excitatrice rectangulaire comme pour une impulsion d'allure gaussienne, à condition de correctement positionner les points de mesure.

Cette évaluation de $S$ suppose :

1. de mesurer le rapport de pentes $K$ sous des conditions $(\alpha, \delta)$ d'excitations qui, pour l'échantillon $\left(S, \tau_{\mathrm{B}}, D\right)$ étudié, correspondent à une excitation suffisamment superficielle (cf. Fig. 6);

2. et d'avoir mesuré par ailleurs $\tau_{\mathrm{B}}$, ou une seconde fonction de $S$ et $\tau_{\mathbf{B}}$. Il faut donc également étudier la décroissance de $\Delta \mathcal{N}(t)$ dans des conditions où cette décroissance obéit à une fonction simple de $S$ et $\tau_{\mathrm{B}}$ (cf. (8) par exemple). Ceci correspond à des temps $t$ de la décroissance de $\Delta \mathcal{N}(t)$ d'autant plus grands que la lumière excitatrice est plus absorbée (cf. Figs. 4 et 5). Pour cette raison : (i) un faisceau lumineux très absorbé $(1 / \alpha \ll W)$, qui se prête bien à la mesure de $K$, se prête mal à l'estimation simultanée de $\tau_{\mathrm{B}}$ et $S$ (cf. cas $\alpha=2,2 \times 10^{3} \mathrm{~cm}^{-1}$, Fig. 5c, par exemple), (ii) un faisceau au contraire très faiblement absorbé $(1 / \alpha>W)$ se prête peu à la mesure de $K$, mais permet l'estimation précise de $\tau\left(\tau_{\mathrm{B}}, S\right)$ (c'est le cas d'un faisceau $1,06 \mu \mathrm{m}, \alpha=30 \mathrm{~cm}^{-1}$, pour un substrat de silicium, cf. Figs. 3a et 5a), et (iii) un faisceau moyennement absorbé est par contre un compromis qui peut donner accès simultanément à $S$ et $\tau_{\mathbf{B}}$ (cf. cas d'une excitation par laser à l'AsGa, $\alpha=330 \mathrm{~cm}^{-1}$, pour des substrats assez minces de silicium, Figs. $4 \mathrm{~b}$ et $6 \mathrm{~b}$ ).

Les expressions et les conditions d'exploitation des distributions de porteurs $\Delta n(x, t)$ et des rapports de pente $K\left(S, \tau_{\mathrm{B}}, D\right)_{\alpha}$, ont été discutés aux $\S \S 2$ et 3 . Les résultats traités dans les figures 1 à 3 et 6 à 8 de ce travail sont directement applicables à la définition et la réalisation de ces différents types de mesure dans des échantillons de silicium. Comme la condition implicite de l'expression de base (3) est que les excès de porteurs mis en jeu restent du domaine des faibles injections, aussi bien les réponses de courant $\Delta I(t)$ que celles de tension $\Delta V(t)$, respectivement fournies par les études de photoconductivité transitoire réali-

Tableau I. - Exemples de valeurs de $S$ et $\tau_{\mathrm{B}}$ obtenues lors du suivi de substrats de silicium soumis à des contraintes technologiques ou d'irradiation.

[Example of $S$ and $\tau_{\mathbf{B}}$ values measured when checking the changes in silicon substrates due to technological and irradiation contraints].

\begin{tabular}{|c|c|c|c|c|}
\hline Fragment & $K_{\text {expe }}(0,69 \mu \mathrm{m})$ & $\begin{array}{c}\tau(1,06 \mu m) \\
\mu \mathrm{s}\end{array}$ & $\begin{array}{c}s \\
\mathrm{~cm} / \mathrm{s}\end{array}$ & $\begin{array}{l}\tau_{\mathrm{B}} \\
\mu \mathrm{s}\end{array}$ \\
\hline $\begin{array}{l}\text { ZF - B - } 10 \\
\text { VI - hydrogéné }\end{array}$ & 0,06 & 2,00 & 235 & 2,07 \\
\hline $\begin{array}{l}\mathrm{ZF}-\mathrm{B}-10 \\
\text { oxydé à } 800^{\circ} \mathrm{C}\end{array}$ & 0,06 & 1,30 & 108 & 1,31 \\
\hline $\begin{array}{l}\mathrm{ZF}-\mathrm{B}-10 \\
\text { oxydé } 1100^{\circ} \mathrm{C}\end{array}$ & $\sim 0,53$ & 0,18 & $\leqslant 10^{3}$ & 0,18 \\
\hline $\begin{array}{l}\mathrm{ZF}-\mathrm{B}-10 \\
\text { recuit à } 900^{\circ} \mathrm{C} \text { s.Ar }\end{array}$ & $\sim 0,13$ & 0,30 & $\sim 10^{2}$ & 0,3 \\
\hline $\begin{array}{l}\text { ZF - B - } 10 \\
900^{\circ} \mathrm{C} \text { s.Ar + irradié }\end{array}$ & 0,13 & 0,57 & 194 & 0,57 \\
\hline $\begin{array}{l}\mathrm{CZ}-\mathrm{B}-10 \\
\text { recuit à } 900^{\circ} \mathrm{C} \text { s.Ar }\end{array}$ & 0,18 & 0,47 & 412 & 0,47 \\
\hline $\begin{array}{l}\text { CZ - B - } 10 \\
900^{\circ} \mathrm{C} \text { s.Ar + irradié }\end{array}$ & $\sim 0,15$ & 0,72 & $\leqslant 10^{3}$ & 0,72 \\
\hline
\end{tabular}


sées sous tension $V_{0}$ ou courant $I_{0}$ constants, se prêtent alors à ces mesures à partir de la relation :

$$
\Delta I(t), \Delta V(t) \propto \Delta \Sigma(t)=\frac{l W}{\alpha} q \mu_{n}\left(1+\frac{\mu_{p}}{\mu_{n}}\right) \frac{\Delta \mathcal{N}(t)}{W}
$$

où $\Delta \Sigma(t) \propto \Delta \mathcal{N}(t)$ est la variation de conductance de la section d'échantillon (aire $l \times \mathfrak{L}$ ) soumise à l'excitation lumineuse. Le tableau I est un exemple de résultats qui ont été obtenus selon cette méthode, lors du suivi de la durée de vie volumique $\tau_{\mathbf{B}}$ de substrats de silicium $(10 \Omega \mathrm{cm}$, dopant B) soumis à différentes étapes technologiques ou contraintes.

\section{Bibliographie}

[1] REBIAI, S., Influence des traitements technologiques sur la qualité et la tenue aux irradiations de la base de photopiles au silicium. Thèse de $3 \mathrm{e}$ Cycle. Toulouse, Décembre 1985.

[2] Tyagi, M. S., Niss, J. F. et van Overstraten, R. L., Solid State Electron. 25 (1982) 411.

[3] Eränen, S. et Blomberg, M., J. Appl. Phys. 58 (1984) 2372.
[4] Boll, M., Tables numériques universelles (Edition Dunod) 1947.

[5] SHOCKLEY, W., Electrons and holes in semiconductors (DV. Nostrand, Company, INC) 1950, 318.

[6] Bielle-Daspet, D., Lagouin, M. et Therez, F., Proc. 5e E.C. Photovoltaïc Conf. (Reidel Ed.), 1983, 131. 